\title{
LA PERFORMANCE DE L'ENSEIGNANT ET LA RELATION D'AFFECTIVITÉ ET D'APPRENTISSAGE DU POINT DE VUE DE PIAGET ET WALLON
}

\section{ARTICLE D'EXAMEN}

DIAS, Adailton di Lauro ${ }^{1}$

DIAS, Adailton di Lauro. La performance de l'enseignant et la relation d'affectivité et d'apprentissage du point de vue de Piaget et wallon. Revista Científica Multidisciplinar Núcleo do Conhecimento. Ano 04, Ed. 07, vol. 09, pp. 64-71. juillet 2019. ISSN: 2448-0959

\section{RÉSUMÉ}

L'étude aborde le thème de la relation d'affectivité et d'apprentissage. II cite l'importance de la performance de l'enseignant dans ce contexte. II aborde cette affectivité, la cognition et la motivation collaborent au développement de l'apprentissage de l'élève par la médiation des enseignants à l'école quotidienne. II est basé sur des théoriciens tels que Piaget et Wallon. II a pour objectif général de comprendre la relation entre l'apprentissage et l'affectivité du point de vue de Piaget et du Wallon. II apporte comme un problème de recherche: Comment l'affectivité, la cognition et la motivation peuvent collaborer dans le développement de l'apprentissage de l'élève avec la médiation de l'enseignant dans la salle de classe? D'après les études réalisées, on considère que, du point de vue de Piaget, dans sa théorie piagétatienne, l'affectivité, la cognition et la motivation sont des conditions nécessaires dans la constitution de l'intelligence et de l'apprentissage significatif. De l'avis de Wallon, selon sa théorie du wallon, pour l'apprentissage, il est nécessaire de

\footnotetext{
${ }^{1}$ Mestrando em Ciências da Educação (Grendal University). Especialista em Língua Inglesa (FIJ). Graduado em Letras (UNEB). Graduado em Português e Inglês pela Universidade Metropolitana de Santos - SP. Professor EBTT Port/Ing - IFRR.
} 
s'intégrer entre les ensembles fonctionnels (affectivité, cognition, motricité et personne). Il est donc nécessaire de souligner l'importance des relations établies avec les aspects émotionnels des élèves dans le processus d'enseignement et d'apprentissage. Et que les relations impliquant l'affectivité peuvent affecter les uns les autres dans votre comportement, vous aider à acquérir des connaissances plus facilement, les rendre plus intelligents, le conduire et les motiver à des actions impliquant l'apprentissage Important. Ainsi, l'affectivité, la cognition et la motivation aident au développement de l'apprentissage des élèves dans la vie scolaire quotidienne.

Mots-clés: affection, apprentissage, enseignant, Piaget, Wallon.

\section{INTRODUCTION}

L'étude traite de la relation entre l'apprentissage et l'affectivité, en soulignant dans ce processus, l'importance de la performance de l'enseignant en classe, démontrant que son état émotionnel, ainsi que celui des élèves reflète positivement ou négativement dans l'apprentissage. II apporte également le thème de l'affectivité, la cognition et la motivation comme facteurs primaires pour le développement et l'apprentissage de l'étudiant, du point de vue de Piaget et Wallon.

Le problème de l'étude cherche à se poser la question suivante : Comment l'affectivité, la cognition et la motivation peuvent-elles collaborer au développement de l'apprentissage de l'élève avec la médiation de l'enseignant en classe ? Pour atteindre l'objectif général qui est configuré dans la compréhension de la relation entre l'apprentissage et l'affectivité du point de vue de Piaget et wallon et aussi pour répondre au problème de cette étude, la recherche bibliographique a été utilisée. Les réflexions sur la relation entre l'apprentissage et le développement des étudiants ont motivé la construction de ce travail. 


\section{L'AFFECTION, LA COGNITION ET LA MOTIVATION COMME FACTEURS PRIMAIRES POUR LE DÉVELOPPEMENT ET L'APPRENTISSAGE DE L'ÉLÈVE}

L'affectivité, la cognition et la motivation sont des facteurs essentiels pour le développement et l'apprentissage des élèves à l'école quotidienne, y compris pour développer l'intelligence, puisque,

(...) sans affection, il n'y aurait pas d'intérêt, pas besoin, pas de motivation; et par conséquent, des questions ou des problèmes ne seraient jamais posés et il n'y aurait pas d'intelligence. L'affectivité est une condition nécessaire dans la constitution du renseignement (PIAGET, 1992, p. 32).

L'éducation occidentale a comme héritage culturel, la déconnexion de l'homme de ses émotions, ses pensées, sa propre réalité, résultant en la formation d'individus faibles de l'esprit, l'énergie et émotionnellement ignorants. II s'agit donc d'une vision fragmentée de l'homme, qui contribue malheureusement à une rupture dans les relations humaines. Cependant, ces dualismes traditionnels de la pensée occidentale présents dans l'histoire de la psychologie traditionnelle ont été confrontés et remis en question par des théories novatrices dans le but d'intégrer, dialectiquement, la cognition et l'affectivité, la raison et l'émotion.

Cependant, l'affectivité est indispensable pour le développement intellectuel des enfants et des adolescents, elle les pousse à agir dans l'objet de la connaissance. "Mais l'affectivité n'est rien sans intelligence, qui leur donne les moyens et clarifie les fins" (PIAGET, 1992, p.70). À cet égard, l'intelligence agit dans l'affectif, considérablement tout au long du processus de l'existence humaine, dans lequel l'organisation quotidienne fournit à la construction active, des éléments favorables à la formation de la personnalité. 
Ainsi, il est possible de comprendre les relations entre affection et intelligence, cela correspond aux émotions qui se tournent vers le monde extérieur, orientée vers le monde des objets d'étude et d'observation. Ce qu'on appelle lui-même l'intelligence, c'est l'attention que l'enfant et l'adolescent commencent à accorder aux composantes du monde qui les entoure, afin d'essayer de comprendre comment les choses fonctionnent pour s'y adapter.

C'est l'interrelation réciproque de ces deux instances, affective et rationnelle, que se produit la formation et le développement de la personnalité, qui est ici comprise comme le produit de la relation qui se construit entre le sujet et l'environnement socioculturel, aboutissant à un être unique, Individuels.

Piaget (1971) avertit que, malgré une nature différente, l'affectivité et la cognition sont inséparables, car dans toutes les actions symboliques et sensées, elles semblent indissociées. II a documenté le comportement efficace de l'action et de la pensée comme un aspect cognitif, à travers les structures mentales, et un aspect affectif, à travers une énergie, qui est l'affectivité.

Vygotsky (2002) comprend l'homme comme un être qui a la capacité de penser, de raisonner, de déduire et d'abstrait, mais aussi comme quelqu'un doté de sentiments, d'émotions, de désirs, d'imagination et de sensibilisation au monde extérieur. Ainsi, vous ne pouvez pas analyser le sentiment et la raison séparément. II est plutôt logique d'analyser l'ensemble de la question, car elle indique des subventions favorables à d'éventuelles solutions aux problèmes existants. Car « il démontre l'existence d'un système dynamique de significations dans lequel affectif s'unissent et intellectuellement » (VYGOTSKY, 2002, p. 7). Cependant, il est indispensable de renforcer le sens des relations que les éducateurs établissent avec les aspects émotionnels des élèves dans le processus d'enseignement et d'apprentissage.

En ce qui concerne les émotions, Wallon (apud GALV-O, 1995) dit qu'il a été depuis le début de la vie; le défend, comme le premier et le plus fort lien entre les individus. 
Au début de son existence, l'enfant devient progressivement un être sociocognitif lors de la construction progressive, une vision unique et particulière de son existence.

Le Wallon (apud GALV-O, 1995) affirme également que l'affectivité apparaît lorsque des éléments symboliques apparaissent, c'est-à-dire qu'elle se manifeste par le contact avec l'autre, indique clairement que l'affectivité implique un ensemble de manifestations qui englobent des sentiments et des émotions.

Dans cette considération, Galvao aborde dans le texte "l'expressivité et les émotions, selon la perspective wallonne" que:

Dès le début, à travers ses gestes impulsifs, contorsions ou spasmes corporels, ainsi que les expressions émotionnelles les plus primitives, comme pleurer ou sourire, le bébé humain mobilise les gens de son entourage dans une sorte de contagion affective. L'adulte interprète, en conséquence, ses valeurs, ses désirs et ses attentes, le sens des expressions émotionnelles du bébé, étant amené à agir selon ses paramètres culturels, ses désirs et ses croyances individuelles, enveloppés dans le climat de contagion propre à ces (GALVôO, 1995, p. 74).

Cependant, de cette façon que le bébé humain doit s'exprimer sont des manifestations d'émotion pure et provoquent des réactions affectives qui les incitent à répondre aux besoins de l'enfant. En ce sens, l'affectivité est le comportement capable d'affecter l'autre, de sorte qu'il le pousse dans l'action. La première forme d'affection réside dans les émotions, dont le rôle est d'unir les individus les uns avec les autres par des réactions organiques et intimes d'une manière globale et indifférenciée.

C'est grâce à cette union que les élèves lorsqu'ils arrivent à l'école se rapportent les uns aux autres et obtiennent des progrès significatifs dans le domaine cognitif. Avec cela, les liens affectifs se développent et l'enseignant joue un rôle important dans le développement des élèves dans le contexte scolaire. 
Pour adhérer au comportement motivant aux aspects affectifs et cognitifs, il convient de mentionner que :

Le développement de l'intelligence permet sans aucun doute de réveiller la motivation par un nombre croissant d'objets ou de situations. Cependant, tout au long de cette évolution, le principe de base demeure le même : l'affectivité est le ressort moteur des actions, et la raison est à son service (LA TAILLE, 1992, p. 65).

La motivation, à son tour, fait partie intégrante du processus d'enseignement et d'apprentissage, est l'un des éléments considérables et essentiels pour apprendre ou accomplir quelque chose. Toutes les actions sont déplacées par une force de motivation. « La motivation est la force motrice de la conduite. C'est la condition interne qui active l'individu et le prédispose à émettre certaines réponses » (CORIA-SABINI, 2004, p. 83).

Cependant, pour apprendre ou enseigner, une force motrice de motivation doit se produire. L'absence de ce quotidien dans la pratique éducative tant chez l'étudiant que dans la direction et dans le corps professoral nuira au développement cognitif des étudiants.

\subsection{L'AFFECTIVITÉ ET SA RELATION AVEC L'APPRENTISSAGE : LA PERFORMANCE DE L'ENSEIGNANT EN CLASSE}

Comprenant l'importance de l'enseignant agissant dans la salle de classe, il est perçu que son état émotionnel, ainsi que celui des élèves reflète dans l'apprentissage. L'enseignant, en montrant de l'affection dans ses actions, motive l'élève à vouloir participer activement à ses cours, percevant l'enseignant comme un ami toujours prêt à l'aider et à lui enseigner.

Pour Oliveira et Chadwick (2001, p. 52), 
L'état émotionnel et affectif des élèves influence leur apprentissage. La motivation est comme un déclencheur qui stimule l'apprentissage et établit les conditions dans lesquelles il se produit. Les émotions jouent un rôle important dans l'apprentissage à n'importe quelle étape de la vie. Mais ils sont particulièrement importants chez les jeunes élèves, car la situation scolaire est assez artificielle par rapport à leur vie et pas très compatible avec leurs préférences.

La pratique d'un enseignant affectif sera de respecter le rythme d'apprentissage de chaque élève, parce qu'il a la sensibilité de percevoir quand ou non de procéder avec le contenu. Un élève peut avoir besoin de plus de temps pour apprendre une certaine discipline que son collègue, mais il peut aussi être plus rapide dans une discipline qui a plus d'affinités.

Comme l'affirme Mendes (2017), il est nécessaire de réfléchir à la pratique pédagogique et à la formation des enseignants. II affirme que la formation continue reflète le rôle de l'éducateur en tant que professionnel, mais cette formation reflète, en particulier, dans ce professionnel en tant que personne, parce que les individus finissent par être influencés par d'autres personnes et par ce qu'ils apprennent dans décisions ou des choix et ce qui transmettra dans l'école de tous les jours. La théorie choisie par cet auteur est wallonne, dont le but principal est d'intégrer les ensembles fonctionnels - l'affectivité, la cognition, la motricité et la personne et aussi l'organisme moyen.

Ainsi, pour Wallon (2007), cela permet de comprendre la personne dans son intégralité, la comprenant non seulement du point de vue de l'un des ensembles, mais aussi de l'intégration continue entre eux. L'étude d'Henri Wallon (1999) met également l'accent sur l'environnement dans lequel la personne est insérée, parce que l'individu et la personne subissent une influence l'un de l'autre. De ce point de vue, le Wallon considère cet élément comme tout aussi important dans son processus de développement. 
Pour que l'enseignant fasse un bon travail, visant à atteindre le plus grand nombre possible d'élèves, il doit utiliser diverses techniques d'enseignement, en adoptant non seulement des œuvres individuelles, mais aussi en couple, en groupe, etc. En outre, il est également important que les enseignants entretiennent de bonnes relations avec les autres enseignants de l'école, afin d'échanger des informations sur les élèves dans chaque domaine de connaissances.

Dans ce contexte, l'enseignant est chargé de promouvoir des moyens qui assurent la motivation, dans lequel il conduira à l'apprentissage. Concernant ce Coria-Sabini adresses:

La motivation a un aspect cyclique. Tout d'abord, il y a un besoin qui se manifeste par une stimulation interne spécifique. Deuxièmement, il existe des actions visant à atteindre un objectif. Une fois cet objectif atteint, le soulagement et la diminution de la tension sont suivis. Ce soulagement est temporaire et après un certain temps le cycle reprend. Un bébé qui pleure se calme après l'alimentation. Cependant, quelques heures plus tard, la faim réapparaît et devrait être remise en souillet (CORIA-SABINI, 2004, p. 84).

En fait, c'est ce qui devrait se passer en classe, l'enseignant doit avoir des stratégies qui motivent l'élève à agir sur l'objet de la connaissance; lorsque l'élève parvient à tirer ses conclusions, il se sentira soulagé. De là est celui qui entre à nouveau l'enseignant apportant de nouvelles problematisations et l'élève ira une fois de plus à la recherche des nouvelles connaissances. Chaque fois que l'élève atteint l'objectif proposé, c'est à l'enseignant de proposer d'autres défis pour que l'élève continue à développer son potentiel.

En ce qui concerne les moyens ou les méthodologies que l'enseignant utilise pour motiver ses élèves ne viennent pas à l'affaire, chacun fonctionne d'une manière différenciée, ce qui importe est de travailler d'une manière que l'élève perçoit l'apprentissage comme une réalisation personnelle. Comme, par exemple, un élève 
effectue une certaine activité qu'il a jugée incapable de l'accomplir, après que l'enseignant lui a offert un toast le mettant au défi de l'exécuter. Avec cela, l'étudiant peut tirer ses conclusions qu'il est capable d'effectuer l'activité sans avoir besoin de récompenses, mais comme une réalisation personnelle.

Cependant, les approches des auteurs cités, comme Piaget, Vygotsky et Wallon, démontrent comment l'émotion aux côtés de la raison joue un rôle essentiel dans l'apprentissage des élèves. Selon eux, le travail de l'enseignant ne peut se limiter à une simple mémorisation, mais à ressentir et à frissonner avec eux. Malheureusement, cette compréhension de la pertinence et du rôle adopté par les émotions dans le processus d'enseignement et d'apprentissage est rare chez les enseignants, puisque l'information qui guide cette idée n'est pas transmise, et toujours en tenant compte que les théories éducatives, telles que le technicité, qui sous-tendent la pratique pédagogique, ne donnent pas toute valeur à cet aspect émotionnel du développement humain.

Saltini cite de nouveaux paradigmes dans l'éducation, parmi eux, il est intéressant de mentionner ce qu'il dit:

II y aura un espace et un temps à l'école où je pourrai mettre en valeur mes camarades de classe, le monde dans lequel je vis, mes idées, mes fantasmes, mes découvertes, mes inventions, mes désirs et mes rêves. Ceux-ci, émergeant de notre noyau, lient et ont un sens dans les objets du monde extérieur, c'est-à-dire que chacun en lui construit son propre lien, créant leurs symboles et valeurs, ainsi que leur signification particulière, et est à travers ces mêmes significations que le sujet crée des liens entre le monde intérieur. De là et avec cela, nous devons respecter et créer une méthodologie pédagogique qui conduit le sujet à son plein développement (SALTINI, 2008, p. 31).

En ce sens, on s'attend à ce que l'école devienne un environnement d'investigation, dans lequel le sujet de ce processus peut exprimer ses formes de pensée, ses doutes 
et ses découvertes, afin qu'elle améliore sa capacité de communication et s'étende, de manière significative, son insertion dans l'espace qui développe le potentiel des étudiants non seulement dans le domaine de la rationalité, mais aussi dans le domaine des émotions.

En bref, Piaget, en plus de prioriser l'importance de la maturation biologique pour l'acquisition de l'apprentissage, a également considéré l'importance de l'interaction pour la construction des connaissances. Vigotsky avait toute sa théorie soutenue dans l'interaction entre l'individu et le milieu. Pour que l'échange entre pairs soit plus efficace, il a souligné le rôle du langage pour l'expression de la pensée. En se concentrant sur la relation entre les domaines affectif et cognitif, wallon a créé la théorie du développement de la personnalité, en soulignant que celle-ci se forme entre l'affectivité et la cognition. En outre, il a souligné que l'affectivité est l'une des phases les plus anciennes du développement.

\section{CONCLUSION}

L'étude met l'accent sur le besoin de l'enseignant de chercher en classe, l'intégration entre

dimensions affectives et cognitives dans le processus d'enseignement et d'apprentissage, puisque l'affectivité et la cognition sont interconnectées. Dans ce contexte, le développement de l'affectivité précise des élaborations menées dans le plan de renseignement, et aussi, le développement du renseignement nécessite des constructions affectives.

L'affectivité est encore un vaste domaine de recherche, il a été remarqué à travers cette étude qu'il est extrêmement important pour le processus éducatif et surtout pour établir une bonne relation entre l'enseignant et l'étudiant.

Malheureusement, au cours de leur développement, les enfants grandissent souvent dans un environnement où les gens ne se respectent pas les uns les autres, vivant dans l'hostilité, où l'affectivité est absente. La tendance est que ces enfants deviennent 
agressifs tant dans leur famille qu'à l'école. À partir du moment où les enfants deviennent agressifs, des difficultés surgiront dans les relations sociales. Par conséquent, il est nécessaire que l'enseignant sache comment traiter avec ses élèves en démontrant de l'affection dans ses actions.

Cependant, l'éducateur doit être conscient qu'il ne suffit pas d'être seulement affectif pour l'élève d'apprendre. Pour cela, l'enseignant doit d'abord être un chercheur, soucieux de sa pratique pédagogique, engagé dans sa fonction sociale et éthique dans ses actions.

L'affectivité n'est qu'un moyen d'améliorer la relation enseignant-élève, ce qui contribuera à la réussite scolaire des deux. C'est par l'affection au moment du dialogue entre tous les membres de la communauté scolaire que les doutes peuvent être résolus, l'indiscipline peut être contournée et le désir de l'élève d'apprendre sera élevé et l'apprentissage atteint.

\section{RÉFÉRENCES}

CORIA-SABINI, Maria Aparecida. Psicologia do Desenvolvimento. 2. ed. São Paulo: Ática. 2004.

GALVÃO, Izabel. Henri Wallon: Uma concepção dialética do desenvolvimento infantil. Petrópolis, RJ: Vozes, 1995.

LA TAILLE, Yves de; OLIVEIRA, Marta Kohl de; DANTAS, Heloysa. Piaget, Vygotsky e Wallon: teorias psicogenéticas em discussão. 13. ed. São Paulo: Summus, 1992.

MENDES, Daniela Barros. Memórias afetivas: A constituição do professor na perspectiva de Henri Wallon. Sao Paulo: Loyola, 2017.

PIAGET, Jean. A equilibração das estruturas cognitivas. Problema central do desenvolvimento. Trad. Álvaro Cabral. Rio de Janeiro: Zahar, 1976. 
. Desenvolvimento e aprendizagem. In: BRINGUIER, J.; PIANCELLA, J.R NESS. J.S.V., Org. In: Studying teaching. 2. ed. Prentice-Hall, 1971.

SALTINI, Cláudio J. P. Afetividade e Inteligência. 5. ed. - Rio de Janeiro: Wak Editora. 2008.

VYGOTSKY, Lev Semenovich. A formação social da mente: o desenvolvimento dos processos psicológicos superiores. 6. ed. São Paulo: Martins Fontes, 2002.

WALLON, Henri. A evolução psicológica da criança. Lisboa: Edições 70, 1999.

Afetividade e aprendizagem - Contribuições de Henry Wallon. São

Paulo: Edições Loyola, 2007.

Soumis : juin 2019.

Approuvé : juillet 2019. 